\title{
Respuesta familiar ante la revelación de la orientación sexual, desde el discurso de hombres homosexuales
}

\author{
Family response to the revelation of the sexual \\ orientation, from the discourse of homosexual men
}

\section{Resposta familiar perante à revelação da orientação sexual, desde o discurso de homens homossexuais}

\author{
P. Rodríguez-Bustamante ${ }^{\mathrm{a} 1^{*}}$, C.J. Mayorquin-Muñoz ${ }^{\mathrm{b} 1}$, F.J. Báez-Hernández ${ }^{\mathrm{c} 2}$, \\ V.Nava-Navarro ${ }^{\mathrm{d} 2}$, E. Rico-Pérez ${ }^{\mathrm{e} 1}$ \\ ORCID: \\ ${ }^{\mathrm{a}} 0000-0003-0551-9947 \quad{ }^{\mathrm{b}} 0000-0001-6415-738 \mathrm{X} \quad{ }^{\mathrm{c}} 0000-0003-4986-6596$ \\ ${ }^{\mathrm{d}} 0000-0002-8670-3510 \quad{ }^{\mathrm{e}} 0000-0003-3852-4793$ \\ ${ }^{1}$ Programa de Maestría en Enfermería, Facultad de Enfermería, Benemérita Universidad Autónoma de Puebla, \\ Puebla, México \\ ${ }^{2}$ Facultad de Enfermería, Benemérita Universidad Autónoma de Puebla. Puebla, México
}

Recibido: 6 noviembre 2018

Aceptado: 15 mayo 2019

\section{Resumen}

Introducción: La homosexualidad representa fragmentar las normas establecidas socialmente, provenientes de una sociedad sectorizada, que adopta un patrón de obligatoriedad heteronormativa, por lo que una orientación sexual disidente conlleva enfrentar un sinfín de prejuicios, con afecciones significativas en el entorno familiar.

Objetivo: Conocer la respuesta del entorno familiar ante la revelación de la orientación sexual, desde el discurso de hombres homosexuales.

Métodos: Estudio cualitativo descriptivo, en el que se realizaron entrevistas semiestructuradas y observación participante a cinco hombres homosexuales de 19 a 36 años de edad de la ciudad de Puebla, México, a través de un muestreo teórico y el empleo de participantes clave para el acceso a los informantes. Resultados: Los hallazgos se estructuraron en torno a una categoría central: respuesta familiar ante un integrante homosexual, de la cual se desprenden tres subcategorías: creencia familiar, conflicto familiar y proceso de pérdida familiar a la heteronormatividad, la cual se encuentra integrada por la agrupación

\footnotetext{
* Autor de correspondencia. Correo electrónico: pavel_brp@hotmail.com https://doi.org/10.22201/eneo.23958421e.2019.3.690 
de múltiples códigos, clasificados de acuerdo a las cinco etapas del proceso de pérdida, propuestas por Kübler-Ross.

Discusión: Las respuestas familiares ante la homosexualidad son dinámicas, sumergidas en un proceso de negación que con el tiempo puede llegar a la aceptación.

Conclusiones: Las respuestas familiares desencadenadas por la homosexualidad se expresan de forma multifacética y a manera de proceso; el conocimiento de éstas puede ayudar a guiar el actuar de enfermería y del equipo de salud, para planear actividades y estrategias ante el proceso de pérdida familiar de la heteronormatividad.

Palabras clave: Relaciones familiares; homosexualidad masculina; investigación cualitativa; enfermería; México.

\section{Abstract}

Introduction: Homosexuality represents a deviation from the social norms established by a sectorial society which imposes having a mandatory heterosexual pattern. Because of this, having a different sexual orientation implies facing prejudices which have diverse impacts on the family environment.

Objective: To know the response of the family environment towards the revelation of the sexual orientation from the perspective of homosexual males.

Methods: This is a qualitative and descriptive study with theoretical sampling and key informing, which used semi-structured interviews and observations on five homosexual males from the city of Puebla, Mexico, whose ages ranged from 19 to 36 years old.

Results: The findings clustered around a central category: the family response towards having a homosexual member, from which three subcategories emerged: family beliefs, family conflict, and the loss of the heterosexual normativity, reflected by multiple codes classified in the five stages of the process of a loss proposed by Kübler-Ross.

Discussion: The family responses towards homosexuality are dynamic and framed by a denial process which eventually can lead to the acceptance.

Conclusions: The family responses towards having a member being homosexual are multiple; thus, an understanding of these responses can help nurses and other health professionals to plan activities and strategies aimed at addressing the process of the family loss of the heterosexual normativity.

Keywords: Family relations; homosexuality male; qualitative research; nursing; Mexico.

\section{Resumo}

Introdução: A homossexualidade representa fragmentar as normas estabelecidas socialmente, provenientes de uma sociedade setorizada, que adopta um padrão de obrigatoriedade heteronormativa, pelo que uma orientação sexual dissidente faz enfrentar uma infinidade de preconceitos, com afecções significativas no entorno familiar.

Objetivo: Conhecer a resposta do entorno familiar perante a revelação da orientação sexual, desde o discurso de homens homossexuais.

Métodos: Estudo qualitativo descritivo, no qual se realizaram entrevistas semiestruturadas e observação participante a cinco homens homossexuais de 19 a 36 anos de idade da cidade de Puebla, México, a través de uma amostragem teórica e o emprego de participantes chave para o acesso aos informantes. Resultados: Os achados estruturaram-se em volta de uma categoria central: resposta familiar frente a um integrante homossexual, da qual se desprendem três subcategorias: crença familiar, conflito familiar e processo de perda familiar à heteronormatividade, a qual se encontra integrada pela agrupação de múltiplos códigos, classificados de acordo com as cinco etapas do processo de perda, propostas por Kübler-Ross. Discussão: As respostas familiares diante da homossexualidade são dinâmicas, submersas em um processo de negação que com o tempo pode chegar à aceitação. 
Conclusões: As respostas familiares desatadas pela homossexualidade expressam-se de forma multifacetada e em forma de processo; o conhecimento destas pode ajudar a guiar o agir da enfermagem e da equipe de saúde, para planejar atividades e estratégias frente ao processo de perda familiar da heteronormatividade. Palavras chave: Relações familiares; homossexualidade masculina; pesquisa qualitativa; enfermagem; México.

\section{Introducción}

La homosexualidad es un término cuyas concepciones son diversas, complejas e indeterminadas, por un lado, puede denotar un desarrollo incorrecto en el ser humano y por consiguiente inadecuado para desempeñar funciones reproductivas ${ }^{1}$. Por otro lado, representa la fragmentación de las normas aceptadas y establecidas socialmente, conlleva enfrentarse a un sinfín de prejuicios, estereotipos, homofobia e inseguridad, como respuesta de una colectividad sectorizada ante una condición rechazada y sancionada moral, religiosa e inclusive legalmente ${ }^{2,3}$.

En este sentido, la homosexualidad crea discusión y controversia, genera inestabilidad y desconcierto a nivel social con afecciones significativas y de mayor impacto en el entorno familiar, resultado de obtener un patrón de obligatoriedad heteronormativa alterada, al complicar y fracturar las estructuras internas, inmersas en un proceso de constantes enfrentamientos y nuevos aprendizajes ${ }^{1}$.

Históricamente, la familia ha sido protectora de las normas que salvaguardan la heterosexualidad y en consecuencia, ha tendido a suprimir a todas aquellas personas que no encuadran ante ese marco sexual ${ }^{4}$. De acuerdo a algunos estudios, se ha encontrado que la familia se fragmenta y trunca su equilibrio ante la homosexualidad ${ }^{1,5}$, sus integrantes generan sentimientos de pérdida, frustración y culpa, manifestaciones de rechazo, desprecio, humillación y discriminación ${ }^{2,3,6-8}$; por esta razón, el manejo de la homosexualidad en algún integrante del grupo familiar resulta confuso para las figuras paternas, pero es igualmente complicado para la persona homosexual, quien enfrenta en directo las experiencias de disgregación y transgresión social, por lo que potencia el daño individual en aquellos con una orientación sexual disidente ${ }^{1,4,9}$.

Ante este panorama, los hombres homosexuales (HH) figurados como población vulnerable, se conducen por la vía de la invisibilidad pública y la de los servicios de salud, de manera que incrementan los riesgos de afectar su salud física, psicológica y social ${ }^{10,11}$, los cuales se acentúan principalmente en el desarrollo de enfermedades mentales y en la adquisición de infecciones de transmisión sexual ${ }^{2,3}$. Escenarios que propician la urgente intervención del personal de enfermería en busca de promover, mantener y mejorar la salud de quienes se encuentran en situaciones de desigualdad y vulnerabilidad ante las oportunidades sociales ${ }^{11}$. De esta manera, la presente investigación tiene como propósito conocer la respuesta del entorno familiar, ante la revelación de la orientación sexual desde el discurso de HH.

\section{Metodología}

Estudio cualitativo, descriptivo ${ }^{12}$, que permitió tener un acercamiento a la respuesta del entorno familiar de $\mathrm{HH}$ ante la revelación de su orientación sexual, en el cual los investigadores desempeñaron un rol de observador participante al cumplir la función de espectadores en el desarrollo de las entrevistas semiestructuradas ${ }^{13}$, llevadas a cabo con una guía de preguntas agrupadas por categorías, mediante éstas los participantes se expresaron de manera libre, espontánea y sin interrupciones ${ }^{14}$.

La selección de los HH se realizó a través de muestreo teórico, el cual se basa en las necesidades de información detectadas en los resultados, hasta que se logró la saturación de datos ${ }^{15}{ }^{16}$; el acceso a los informantes se desarrolló por medio de dos participantes clave (PC), para facilitar el proceso de reclutamiento, a quienes se explicó el objetivo, la metodología y los criterios de inclusión que consistieron en HH residentes del municipio de Puebla, México, mayores de 18 años y que hayan externado su orientación sexual a la familia. Los PC hicieron extensiva la invitación y se concretaron citas individuales, se 
realizaron las entrevistas semiestructuradas, en un área privada que permitió mantener el anonimato, en un ambiente de tranquilidad y respeto.

Los datos se recabaron mediante una grabadora de voz digital marca Sony Icdpx333, las entrevistas semiestructuradas fueron transcritas en Microsoft Office Word 2010, se obtuvo un total de 84 cuartillas. El análisis de datos se llevó a cabo con la propuesta de Rodríguez, Gil y García ${ }^{17}$, la cual consiste en: 1) reducción de datos, mediante codificación abierta, axial y selectiva ${ }^{18}$; 2) disposición y transformación de datos, a través de diagramas para facilitar la comprensión de la información; y 3) obtención de resultados y verificación de conclusiones, al contrastar los resultados del estudio con otras investigaciones.

Se realizó triangulación de datos ${ }^{19}$, mediante la integración de los diversos discursos obtenidos, acordes a la singularidad de cada uno de los participantes. Se llevó a cabo una descripción y mapas conceptuales de los hallazgos, se identificaron coincidencias y se contrastaron con las etapas del proceso de pérdida propuestas por Kübler-Ross ${ }^{20}$, lo que permitió mayor riqueza interpretativa y analítica de los hallazgos del estudio ${ }^{16}$.

Durante el análisis de datos se consideraron los criterios de calidad propuestos por Lincoln y Guba ${ }^{21}$. que consisten en: 1) credibilidad, dado que se realizó transcripción textual del total de entrevistas semiestructuradas con puntuaciones detalladas en gestos y expresiones corporales; 2) transferibilidad, puesto que el reclutamiento se basó en un muestreo teórico, con criterios específicos de selección; 3) dependencia, ya que se identificó y describió con minuciosidad a los participantes, así como la técnica de recolección y análisis de datos; por último, 4) confirmabilidad, debido a que la información se recabó sin inferencias particulares y de manera inductiva. El estudio se apegó al Reglamento de la Ley General de Salud en Materia de Investigación, se obtuvo consentimiento informado, se conservó el anonimato, la confidencialidad de los datos recabados y se respetó la integridad de los participantes ${ }^{22}$.

\section{Resultados}

Se realizaron cinco entrevistas semiestructuradas a hombres homosexuales de entre 19 y 36 años de edad, el estado civil de cuatro de ellos fue soltero y uno en unión libre, la ocupación de tres participantes fue estudiante y dos de ellos empleados, todos pertenecientes a familias nucleares de origen. El análisis de datos permitió identificar una categoría central: respuesta familiar ante un integrante homosexual, de la cual desprenden tres subcategorías con sus respectivos códigos vivos.

De acuerdo al discurso expresado por los $\mathrm{HH}$, la primera subcategoría identificada fue creencia familiar, concebida como la idea o pensamiento que se asume como verdadero ${ }^{23}$, expresado a través de actitudes de sospecha familiar ante la homosexualidad:

Se empezó a dar cuenta de eso (...) tu manera de correr es diferente, de hablar o tus gestos ya te empiezan a ser más (...) empiezan a ser diferentes a o sea, bueno de los niños normales, porque de los niños normales son como muy rectos, muy rectos (...) muy hasta sus gestos, sus ademanes son firmes no son como es (...) firmes, y entonces se dan cuenta ¿no?, y ahí empezó mi problema $(. .).(\mathrm{p} 3$; entrevistas con discursos similares: 1, 2, 4, 5).

Básicamente como que yo ya daba muestras de mi orientación. Entonces, así como que a veces te sale movimiento o una sonrisita, una mueca y dicen ah pues eso no es normal ¿no?, (...) si sabían pero no querían externarlo en forma completa igual que yo $(\ldots)$ (p4; entrevistas con discursos similares: $1,2,3,5)$.

La segunda subcategoría se denominó conflicto familiar, entendida como aquella situación de desacuerdo u oposición, resultado de un acto de incompatibilidad ${ }^{24}$, dentro de las normas familiares. El discurso de los participantes permitió identificar que es desencadenada a través de la revelación de la homosexualidad:

(...) le dije a mi papá este (...) ‘ ¡ah y si lo pensabas te lo confirmo!’ (...) y le empecé así como a manera de ofensa para mí, (...) 'y si lo pensabas, te lo confirmo ;soy puto!’ [risas], (...) cuando yo le dije se enojó (p3; entrevistas con discursos similares: $1,2,4,5)$.

Respuesta familiar ante la revelación de la orientación sexual • P. Rodríguez-Bustamante et al. 
(...) ya no me gustaba decirle mentiras a mi mamá porque yo salía y pues me iba con mi pareja y así, entonces llegó un punto en el que ya no quería mentirle más y le dije pues que tenía una relación, pero que era con un hombre y así, (...) su reacción fue como que negativa, porque ella decía que no era lo normal (...) (p2; entrevistas con discursos similares: $1,3,4,5)$.

Asimismo, se encontró que en la mayoría de los participantes la primera figura en recibir la revelación de la orientación homosexual fue la madre:

(...) dije 'pues ya es tiempo de que mis papás lo sepan’, (...) pues ya borracho, mi mamá igual había tomado algunas copas (...) y ay dije 'mira ¿sabes qué? él es mi novio’, dije ‘ya, ya me cansé de estarlo ocultando!’, él ya había ido varias veces (p1; entrevistas con discursos similares: 2, 4, 5).

(...) yo decido hablar primero con mi mamá, (...) le conté a mi mamá y mi mamá al millón, su cara fue de que ‘ay hijo’ pues ya literal (...) con su cara y su reacción me dijo ‘ay hijo pues yo la verdad ya sabía desde hace (...) cuídate mucho y no quiero que andes de vestida (p5; entrevistas con discursos similares: 1, 2, 4).

La tercera subcategoría se designó proceso de pérdida familiar a la heteronormatividad, entendida como la serie de reacciones desencadenadas ante la pérdida de la heterosexualidad en algún integrante ${ }^{25}$, misma que a través de codificación selectiva ${ }^{18}$, se agrupó de acuerdo a la propuesta teórica de Kübler-Ross ${ }^{20}$, quien propone cinco etapas integradas por respuestas a las pérdidas en los seres humanos, entendidas como la secuencia de reacciones emocionales que permiten protegerse ante amenazas desencadenadas frente a la pérdida, que incluye cambios físicos y emocionales con afecciones individuales y sociales ${ }^{26,27 .}$

\section{Etapa de negación}

La primera etapa del proceso se define como la imposibilidad de aceptar y reconocer ${ }^{27}$ la homosexualidad de un integrante, misma que de acuerdo al discurso de los participantes, puede ser exteriorizada a través de comportamientos como el silencio, la evasión y la incredulidad familiar:

Silencio familiar: (...) y mi papá se quedó así de ¡ah bueno!, no me dijo nada, pues todos se callaron y yo así de (...) [expresión de duda] (p1). (...) entonces en ese, entra mi papá y ve a mi pareja que me está abrazando y no, no dijo nada en ese momento (...) (p4).

Evasión familiar: Ajá, le dije ya hablando bien así le dije, 'es que yo soy gay' y nomás me dijo 'no, no te quiero escuchar' así como que (...) (p3). (...) cuando trataba como de decirme de eso yo tampoco no quería decirle nada, o sea era como de que no quería intensificar más la situación, entonces como le empezaba hablar de otras cosas o le decía que no quería platicar en ese momento (...) como que evadía el problema [risas] (p2).

Incredulidad familiar: (...) a veces mi mamá me peguntaba así como si realmente me gustaban los hombres o si me gustaban las mujeres o si este, alguna vez me llegarían a gustar o cosas así, porque pues las mamás tienen esa mentalidad de que uno se va a ir con una mujer, va a tener una familia, hijos y cosas así ¿no? (p2; entrevistas con discursos similares: $1,3,5)$. (...) mi hermano el que va después de mí (...), le dije 'oye pues es que pasa esto' y me dijo 'no, te entiendo, sé que me habías comentado pero yo cré que era mentira', (... y le dije 'no pues o sea, si es verdad', le dije 'o sea soy así y quiero que me aceptes así' (p1; entrevistas con discursos similares: 2, 3, 5).

\section{Etapa de ira}

Se define como la reacción emocional y comportamental de rabia y hostilidad ${ }^{27}$ dirigida al integrante homosexual, la cual se manifestó a través de la violencia familiar: Era muy ofensivo, cuando yo era niño le molestaba verme y me decía ‘que joto para acá y joto para allá, me decía ‘ipor qué caminas así? pinche joto’ y esto, así pues (...) y a veces se burlaba de mi enfrente de sus amigos, era muy (...) muy mendigo mi hermano (...) (p5; entrevistas con discursos similares: $1,3,4)$. (...) entonces hubo una ocasión, de hecho fue en año nuevo que empezaron a hablar mal de mí, mis primos, que pinche puto y cosas así (...)” (p1; entrevistas con discursos similares: 3, 4, 5). Por parte de mi papá, sí igual, cuando hacia movimientos que no le gustaban (...) si llegaba a los golpes” (p4; entrevistas con discursos similares: 1, 3, 5). 


\section{Etapa de negociación}

Se concibe como una forma de afrontar los cambios suscitados con el compromiso de intentar recuperar lo perdido ${ }^{27}$ en la relación familiar, fue identificada a través de la conciliación familiar: (...) con mi mamá sí, como que no nos hablábamos los primeros días ni nada y ya después pues fue desapareciendo eso (...) o sea como que ella tuvo la (...) este, dio el paso como para que siguiéramos bien y yo dije ¡ah pues yo también! (p2; entrevistas con discursos similares: 1, 3, 4, 5). (...) mi papá después si me trataba de hablar bien, o sea, (...) él me decía 'mira es que las cosas no son así' $y$ me empezaba hablar bonito (...) (p3; entrevistas con discursos similares: 1, 2, 4, 5).

\section{Etapa de depresión}

Es entendida como la sensación de pérdida y vacío de la heteronormatividad de algún integrante en la familia, la cual se manifiesta a través de expresiones de tristeza y dolor, como resultado del cambio evidente ${ }^{27,28}$, identificada a través de la aflicción familiar: Mi madre también, ella me dijo 'mejor es que te vayas', sí hubo dolor, al final de cuentas si ella (...) si ya sabían, y que básicamente era chistoso porque, (...) ya cuando se hace abierto, así de sabes que este (...) pues mejor no, mejor vete (p4; entrevistas con discursos similares: 2, 5). (...) mi hermana dijo 'no pues, puedes dejar de ser mi hermano ¿si quieres?' me dijo, '¿quieres que deje de ser tu hermano? entonces ojalá nunca te arrepientas' le dije, y ya me dice 'no, pero que no sé qué...' (...) al último este (...) se le soltaron las lagrimillas (...) (p5; entrevistas con discursos similares: 2, 4).

\section{Etapa de aceptación}

La quinta y última etapa del proceso, se refiere al reconocimiento de la pérdida tras el balance y la asignación de nuevos significados que toma la familia, con una actitud de responsabilidad ${ }^{27}$ ante el integrante homosexual. Identificada a través del apoyo familiar, expresada por los siguientes discursos: (...) me dijo 'pues eres mi hermano, entonces no te voy a juzgar, si a ti te gusta eso pues (...) está bien, estoy contigo, ya ves (...) papá, mamá también pues te apoyan, ¿por qué yo no?'y dije ok (p1; entrevistas con discursos similares: 2, 4, 5). Emm (...) sí, porque pues ya llegó el momento donde pues ya empezaba a tener parejita, y pues ya todos iban a verme y pues yo también quería ver como lo iban a tomar, si se iban a ofender (...) y no al contrario, lo tomaron muy bien, los niños hasta tío le decían a mi ex y así (...) y todo muy padre (p5; entrevistas: 1, 2, 4). (Ver Figura 1).

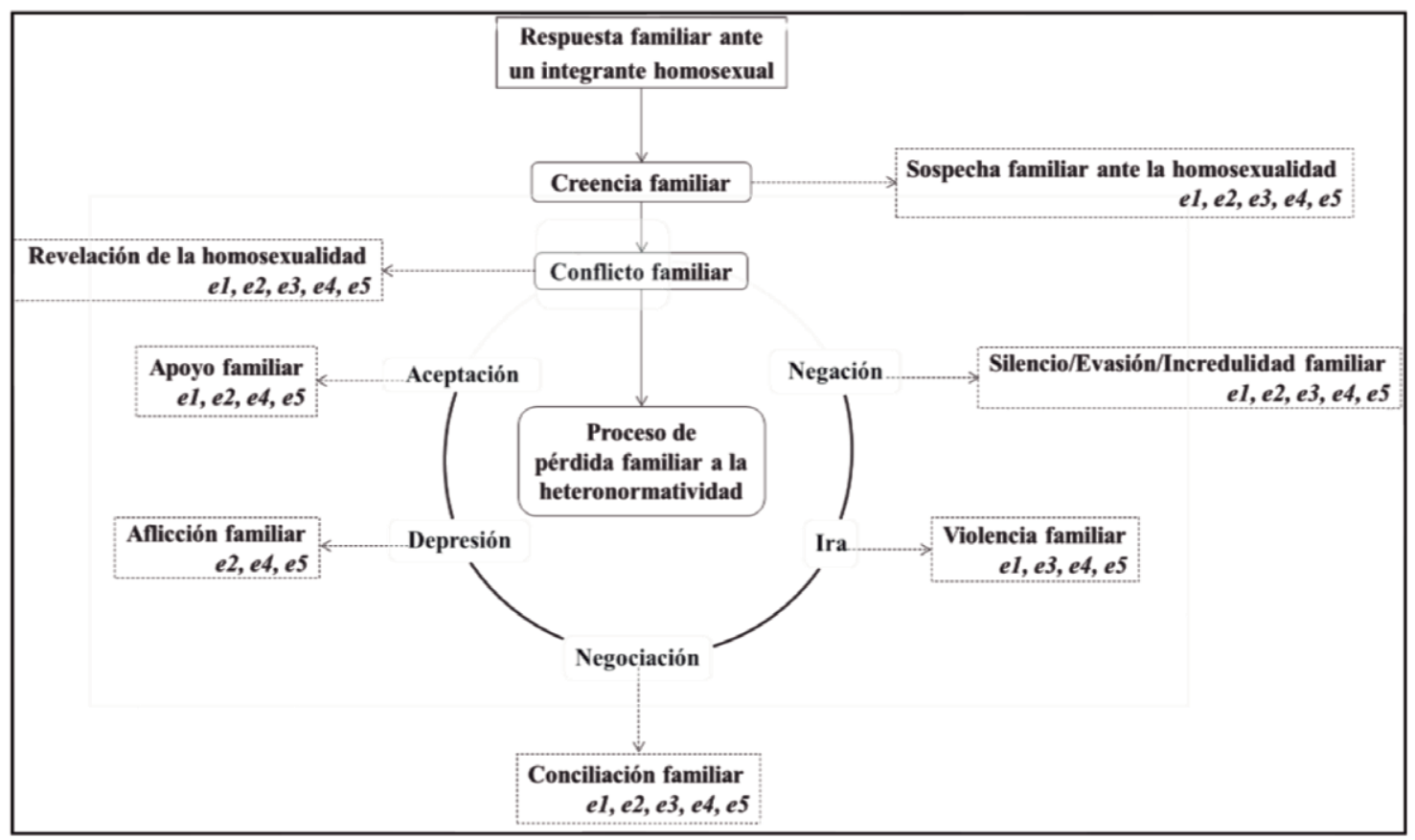

Figura 1. Respuesta familiar ante un integrante homosexual 


\section{Discusión}

El presente estudio permitió identificar a través del discurso de los HH la respuesta familiar ante la revelación de la orientación sexual de uno de sus integrantes, en donde los resultados, al presentarse como una secuencia de reacciones emocionales permitieron retomar la propuesta teórica de Kübler-Ross ${ }^{20}$. Como manifestación inicial se encontró que todos los HH recibieron expresiones de duda y sospecha referente a su sexualidad, lo cual coincide con lo referido por List ${ }^{8}$ y Ceballos-Fernández ${ }^{1}$, ya que las expresiones corporales de los $\mathrm{HH}$ no figuran como representaciones masculinas y generan suspicacia de su orientación sexual, en donde la familia expresa rechazo ante el desafío de la norma heterosexual.

Otro resultado identificado fue el de la subcategoría conflicto familiar, el cual fue desencadenado por la revelación de la orientación sexual, mismo que genera cambios en el sistema familiar de los $\mathrm{HH}$, por lo que se encontraron coincidencias con Zambrano, Ceballos y Ojeda ${ }^{2}$, González y Toro ${ }^{3}$, Luján y Tamarit $^{6}$, Guarnero ${ }^{7}$ y List ${ }^{8}$, quienes afirman que las familias tienen dificultad para reconocer y apoyar la homosexualidad, producto de la fragmentación de las normas aceptadas y establecidas socialmente. Aunque el grupo familiar asume la orientación homosexual del integrante, sin haber hablado de ella, la exteriorización y confirmación de la misma conlleva una respuesta negativa, con sentimientos de pérdida, frustración y culpa, manifestaciones de rechazo, desprecio, humillación y discriminación. En este contexto, la mayor parte de los participantes reveló su orientación sexual en primer lugar a la figura materna, resultado que presentó similitud con lo que afirma Jiménez y Romero ${ }^{5}$, y Luján y Tamarit ${ }^{6}$, quienes señalan que las madres tienen mayor probabilidad de recibir la noticia antes que la figura paterna.

En relación a la subcategoría proceso de perdida familiar a la heteronormatividad, se encontraron similitudes con algunos estudios, puesto que Jiménez y Romero ${ }^{5}$, Luján y Tamarit ${ }^{6}$, y List $^{8}$ reportan que la homosexualidad de un hijo no suele representar una ruptura definitiva, dado que la familia se encuentra inmersa en un proceso con una serie de estrategias de negación que con el paso del tiempo pueden llegar a la aceptación, lo cual puede establecer una salida saludable, o bien, con la probabilidad de que el proceso se complique y este nunca sea finalizado, como lo reconocen Liz y Avendaño ${ }^{26}$. Es por ello, que el proceso de pérdida familiar a la heteronormatividad es dinámico, su tránsito y duración resulta diverso pero con predominante tendencia a la negación, concordante a lo referido por Ceballos-Fernán$\mathrm{dez}^{1}$, quien menciona que los padres y madres reaccionan de maneras múltiples y negativas al conocer la homosexualidad de sus hijos, lo cual afirman Jiménez y Romero ${ }^{5}$, quienes reconocen que la negación es un intento de rehusarse a la realidad para permitir continuar una relación de cordialidad familiar con el integrante homosexual. Finalmente, el paso del tiempo permite la adopción de estados familiares más positivos en donde la mayoría de los integrantes suele llegar a la aceptación, como lo expuesto por Lujan y Tamarit ${ }^{6}$, quienes sostienen que con el curso de los días la mayoría de las familias adopta sentimientos y expresiones más positivos en comparación a las respuestas iniciales de rechazo dirigidas al HH.

\section{Conclusión}

Los resultados de este estudio permiten sugerir que las respuestas familiares desencadenadas por la revelación de la homosexualidad de algún integrante hombre, se expresan de forma diversa, multifacética y a manera de proceso, el cual puede iniciar en cualquiera de las primeras cuatro etapas (negación, ira, negociación y depresión), hasta llegar a la aceptación y asignar un cierre saludable, o bien, que el proceso traslape, se ancle y nunca sea finalizado.

Se considera que el rechazo a los HH en el entorno familiar es un grave problema, por lo que los hallazgos encontrados pueden ayudar a las enfermeras y a los integrantes del equipo de salud a conocer los probables mecanismos de respuesta familiar desencadenados por la homosexualidad, identificar las posibles situaciones a las que esté haciendo frente el $\mathrm{HH}$, lo que permitirá guiar su actuar profesional para clarificar sus intervenciones y planear actividades oportunas en atención a las necesidades de este grupo vulnerable, considerar estrategias ante el proceso que desde la perspectiva del $\mathrm{HH}$, el grupo familiar percibe como pérdida de la heteronormatividad. 
Finalmente, con base en los resultados presentados, se cree necesario seguir en la profundización del tema y fomentar la realización de estudios en familias de integrantes con orientaciones sexuales disidentes, desde otros abordajes teóricos, con la intención de sensibilizar, derribar prejuicios, comprender y mejorar la aceptación de los HH, para lograr visibilizar su necesaria y pertinente atención ante los servicios de salud.

\section{Responsabilidades éticas}

Protección de personas y animales. En esta investigación no se realizaron experimentos en seres humanos ni en animales.

Confidencialidad de los datos. Se aseguró y en el estudio no aparecen datos que permitan la identificación de los participantes.

Derecho a la privacidad y consentimiento. se obtuvo consentimiento informado, se conservó el anonimato, y se respetó la integridad de los participantes.

Financiamiento. Ninguno.

Conflicto de intereses. Los autores declaran no tener conflicto de intereses.

\section{Referencias}

1. Ceballos-Fernández M. Identidad homosexual y contexto familiar heteroparental: implicaciones educativas para la subversión social. Rev.latinoam.cienc.soc.niñez.juv. 2014; 12(2): 643-58. https://doi.org/10.11600/1692715x.1229140514

2. Zambrano-Guerrero CA, Ceballos-Mora AK, Ojeda-Rosero DE. Reconocimiento de la orientación sexual homosexual. Psicoespacios. 2017; 11(19): 77-93. https://doi.org/10.25057/21452776.940

3. González-Guadarrama J, Toro-Alfonso J. El significado de la experiencia de la aceptación de la orientación sexual homosexual desde la memoria de un grupo de hombres adultos puertorriqueños. Eureka (Asunción) en línea. 2012; 9(2): 158-70. https://bit.ly/2PPGe9c

4. Silva-Luévanos BE. Efectos en el afrontamiento y soporte social ante la revelación de la homosexualidad a la familia: estudio comparativo en gais y lesbianas. Psicogente. 2018; 21 (40): 321-36. https://doi.org/10.17081/psico.21.40.3077

5. Jiménez-Solorzano A, Romero-Mendoza M. “Salir del clóset” en la Ciudad de México. Salud ment. (Méx). 2014; 37(5): 391-97. https://bit.ly/2SbkWnV

6. Luján-Enriquez I, Tamarit-Rovira AM. Dinámica familiar ante la revelación de la orientación homosexual de los hijos/as. INFAD. 2012; 3(1): 301-8. https://bit.ly/2q8PfyZ

7. Guarnero PA. Family and community influences on the social and sexual lives of latino gay men. J. transcult. nurs. 2007; 18(1): 12-8. http://dx.doi.org/10.1177/1043659606294195

8. List-Reyes M. Hablo por mi diferencia. De la identidad gay al reconocimiento de lo queer. México: EÓN; 2009.

9. González-Ortuño G. Teorías de la disidencia sexual: de contextos populares a usos elitistas. La teoría queer en América Latina frente a las y los pensadores de disidencia sexo genérica. De raíz divers. Rev. espec. estud. Latinoam. 2016; 3(5): 179-200. http://dx.doi.org/10.22201/ppela.24487988e.2016.5.58507

10. Moral-De la Rubia J, Valdez-Montero C, Onofre-Rodríguez DJ. Propiedades psicométricas del cuestionario de influencias situacionales para conducta sexual en hombres que tienen sexo con hombres. Rev Mex Invest Psic. 2016; 8 (1). https://bit.ly/2HGaroo

11. Valdez-Montero C, Castillo-Arcos L, Olvera-Franco A, Onofre-Rodríguez DJ, Caudillo-Ortega L. Reflexión de los determinantes sociales de la conducta sexual en hombres que tienen relaciones sexuales con hombres. Cuid. enferm. educ. salud. 2015; 2(1): 34-47.

https://doi.org/10.15443/ceyes.v2i1.650 
12. Rivadeneira-Rodríguez EM. Comprensión teórica y proceso metodológico de la investigación cualitativa. In Crescendo (Chimbote). 2015; 6(2): 169-83. https://bit.ly/2wbyFkS

13. Álvarez-Gayou JL. Cómo hacer investigación cualitativa. Fundamentos y metodología. México: Paidós Educador; 2003.

14. Díaz-Bravo L, Torruco-García U, Martínez-Hernández M, Varela-Ruiz M. La entrevista, recurso flexible y dinámico. Inv Ed Med. 2013; 2(7): 162-7. https://bit.ly/2O0lt8T

15. Polit DF, Hungler BP. Investigación científica en ciencias de la salud. $6^{a}$ ed. México: McGraw Hill Interamericana; 2000.

16. Hernández R, Fernández C, Baptista P. Metodología de la investigación. $6^{a}$ ed. México: McGraw Hill Interamericana; 2014.

17. Rodríguez G, Gil J, García E. Metodología de la investigación cualitativa. Málaga: Aljibe; 1999.

18. Hernández-Carrera RM. La investigación cualitativa a través de entrevistas: su análisis mediante la teoría fundamentada. Cuest. pedagóg. 2014; 23: 187-210. https://bit.ly/2xUr0GO

19. Banister P, Burman E, Parker I, Taylor M, Tindall C. Métodos cualitativos en psicología. México: La Noche Editores; 2004.

20. Kübler-Ross E, Kessler D. On grief and grieving. Finding the meaning of grief through the five stages of loss. New York: Scribner; 2005.

21. Lincoln YS, Guba EG. Naturalistic inquiri. Newbury Park: Sage; 1985.

22. Secretaría de Salud. Reglamento de la Ley General de Salud en Materia de Investigación para la Salud. México: Secretaría de Salud. 1984. https://bit.ly/1SBpqPT

23. Oxford University Press. Oxford spanish dictionary. Reino Unido: Oxford University Press. 2018. https://bit.ly/2ysmqCg

24. Pino-Montoya JW. Los conflictos y la dinámica interna familiar. Memorias. 2012; 10(18): 159-70. https://bit.ly/2ScTqX7

25. Castañeda M. La experiencia homosexual. Para comprender la homosexualidad desde dentro y desde fuera. México: Paidós; 2011.

26. Liz-Bernal R, Avendaño BL. Diseño y análisis psicométrico del inventario IPDP para evaluar el proceso de duelo patológico en adultos. Divers.: Perspect. Psicol. 2018; 14(1): 121-36.

http://dx.doi.org/10.15332/s1794-998.2018.0001.09

27. Miaja-Ávila M, Moral-De la Rubia J. El significado psicológico de las cinco fases del duelo propuestas por Kübler-Ross mediante las redes semánticas naturales. Psicooncología. 2013; 10(1): 109-130. http://dx.doi.org/10.5209/rev_PSIC.2013.v10.41951

28. Moral-De la Rubia J, Miaja-Ávila M. Contraste empírico del modelo de cinco fases de duelo de Kübler-Ross en mujeres con cáncer. Pensam. psicol. 2015; 13(1): 7-25.

http://dx.doi.org/10.11144/Javerianacali.PPSI13-1.cemc 